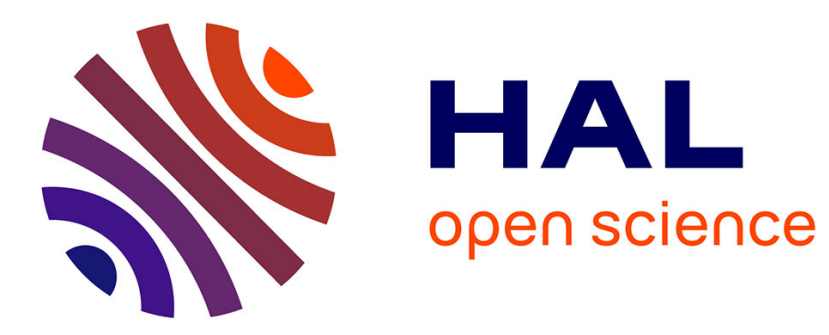

\title{
A Zone of Quiet Based Approach to Integrated Active Noise Control and Noise Reduction for Speech Enhancement in Hearing Aids
}

Romain Serizel, Marc Moonen, Jan Wouters, Søren Holdt Jensen

\section{- To cite this version:}

Romain Serizel, Marc Moonen, Jan Wouters, Søren Holdt Jensen. A Zone of Quiet Based Approach to Integrated Active Noise Control and Noise Reduction for Speech Enhancement in Hearing Aids. IEEE Transactions on Audio, Speech and Language Processing, 2012, 20 (6), pp.1685 - 1697. hal-01393939

\section{HAL Id: hal-01393939 \\ https://hal.science/hal-01393939}

Submitted on 9 Nov 2016

HAL is a multi-disciplinary open access archive for the deposit and dissemination of scientific research documents, whether they are published or not. The documents may come from teaching and research institutions in France or abroad, or from public or private research centers.
L'archive ouverte pluridisciplinaire HAL, est destinée au dépôt et à la diffusion de documents scientifiques de niveau recherche, publiés ou non, émanant des établissements d'enseignement et de recherche français ou étrangers, des laboratoires publics ou privés. 


\title{
A Zone of Quiet Based Approach to Integrated Active Noise Control and Noise Reduction for Speech Enhancement in Hearing Aids ${ }^{1}$
}

\author{
Romain Serizel2 ${ }^{2}$, Marc Moonen ${ }^{2}$, \\ Jan Wouters ${ }^{3}$ and Søren Holdt Jensen ${ }^{4}$
}

August 2012

Published in The IEEE Transactions on Audio, Speech and Language Processing, Vol. 20, No. 6, Aug 2012, pp 1685-1697

\footnotetext{
${ }^{1}$ This report is available by anonymous ftp from ftp.esat.kuleuven.be in the directory $\mathrm{pub} /$ sista/rserizel/reports/11-43.pdf

${ }^{2}$ K.U.Leuven, Dept. of Electrical Engineering (ESAT), Research group SCD (SISTA) Kasteelpark Arenberg 10, 3001 Leuven, Belgium, Tel. +32 1632 9607, Fax +32 16 321970, E-mail: romain.serizel@esat.kuleuven.be. This research work was carried out at the ESAT laboratory of the Katholieke Universiteit Leuven, in the frame of the Marie-Curie Fellowship EST-SIGNAL program (http://est-signal.i3s.unice.fr) under contract No. MEST-CT-2005-021175, and the Concerted Research Action GOA-AMBioRICS. The scientific responsibility is assumed by its authors.

${ }^{3}$ Katholieke Universiteit Leuven, Department of Neurosciences, ExpORL, O. \& N2, Herestraat 49/721, 3000 Leuven, Belgium, E-mail: Jan.Wouters@med.kuleuven.be

${ }^{4}$ Aalborg University, Department of Electronic Systems, MISP, Niels Jernes Vej 12 A6-3, 9220 Aalborg, Denmark, E-mail: shj@es.aau.dk
} 


\begin{abstract}
This paper focuses on speech enhancement in hearing aids and presents an integrated approach to active noise control and noise reduction which is based on an optimization over a zone of quiet generated by the active noise control. A basic integrated active noise control and noise reduction scheme has been introduced previously to tackle secondary path effects and effects of noise leakage through an open fitting. This scheme however, only takes the sound pressure at the ear canal microphone into account. For an integrated active noise control and noise reduction scheme to be efficient, it is desired to achieve active noise control at the eardrum which in practice is away from the ear canal microphone. In some cases it can also be desired to achieve noise control over a zone not limited to a single point.

Two different schemes are presented. A first scheme based on a mean squared error criterion expressed at a remote point away from the ear canal microphone and a second scheme based on an average mean squared error criterion over a desired zone of quiet. They are both compared experimentally with the original scheme for both active noise control and integrated active noise control and noise reduction, respectively.
\end{abstract}




\title{
A Zone-of-Quiet Based Approach to Integrated Active Noise Control and Noise Reduction for Speech Enhancement in Hearing Aids
}

\author{
Romain Serizel, Marc Moonen, Jan Wouters and Søren Holdt Jensen
}

\begin{abstract}
This paper focuses on speech enhancement in hearing aids and presents an integrated approach to active noise control and noise reduction which is based on an optimisation over a zone-of-quiet generated by the active noise control. A basic integrated active noise control and noise reduction scheme has been introduced previously to tackle secondary path effects and effects of noise leakage through an open fitting. This scheme however, only takes the sound pressure at the ear canal microphone into account. For an integrated active noise control and noise reduction scheme to be efficient, it is desired to achieve active noise control at the eardrum which in practice is away from the ear canal microphone. In some cases it can also be desired to achieve noise control over a zone not limited to a single point.

Two different schemes are presented. The first scheme is based on a mean squared error criterion expressed at a RP away from the ear canal microphone and the second scheme is based on an average mean squared error criterion over a desired zone-of-quiet. They are both compared experimentally with the original scheme for both active noise control and integrated active noise control and noise reduction, respectively. The remotepoint approach then allows to restore the performance of the original scheme at the desired remote point while the zone-ofquiet approach allows to increase performance up to $3 \mathrm{~dB}$ on the desired zone-of-quiet.
\end{abstract}

Index Terms-Hearing aids, noise reduction, multichannel Wiener filter, active noise control, zone-of-quiet

\section{INTRODUCTION}

State-of-the-art hearing aids perform Noise Reduction (NR) in order to improve their output Signal-to-Noise Ratio (SNR) and hence to allow for a better speech understanding in background noise [1]. Conventional NR systems such as the

Copyright (c) 2010 IEEE. Personal use of this material is permitted. However, permission to use this material for any other purposes must be obtained from the IEEE by sending a request to pubs-permissions@ieee.org.

This research work was carried out at the ESAT Laboratory of Katholieke Universiteit Leuven, in the frame of K.U.Leuven Research Council $\mathrm{CoE}$ EF/05/006 Optimization in Engineering (OPTEC), PFV/10/002 (OPTEC), Concerted Research Action GOA-MaNet, the Belgian Programme on Interuniversity Attraction Poles initiated by the Belgian Federal Science Policy Office IUAP P6/04 (DYSCO, 'Dynamical systems, control and optimization', 20072011), Research Project IBBT, Research Project FWO nr. G.0600.08 ('Signal processing and network design for wireless acoustic sensor networks'), EC-FP6 project SIGNAL: 'Core Signal Processing Training Program'. The scientific responsibility is assumed by its authors.

R. Serizel and M. Moonen are with the Department of Electrical Engineering, Katholieke Universiteit Leuven, ESAT-SCD, Kasteelpark Arenberg 10, B-3001 Leuven, Belgium.

J. Wouters is with the Division of Experimental Otorhinolaryngology, Katholieke Universiteit Leuven, ExpORL, O.\& N2, Herestraat 49/721, B-3000 Leuven, Belgium.

S.H. Jensen is with the Department of Electronic Systems, Aalborg University, Niels Jernes Vej 12, DK-9220 Aalborg, Denmark.
Generalised Sidelobe Canceller (GSC) [2] or techniques based on the Multichannel Wiener Filter (MWF) [3] are commonly used.

With the advent of efficient feedback control schemes [4][5], the usage of hearing aids with an open fitting has become more common. Whereas removing the earmold reduces the occlusion effect and improves the physical comfort [6], one major drawback is that the direct signal leakage through the fitting into the ear canal cannot be neglected anymore. Conventional NR does not take this contribution into account. Combined with the attenuation in the acoustic path between the hearing aid loudspeaker and the eardrum (the so-called secondary path), the noise leaking through the fitting can then override the action of the NR.

One efficient way to cancel this undesired noise leakage is to use Active Noise Control (ANC) [7][8]. In the hearing aids framework, ANC then has to be performed together with the NR. A scheme integrating the two functional blocks based on a filtered-X [9][10][11] version of the MWF (the socalled FxMWF) has been introduced in [12]. The objectives of this scheme are to attenuate the noise component of the leakage (i.e. ANC) and to minimise the difference between an unknown desired speech signal and the signal delivered at the eardrum (i.e. NR). In practice however, the ear canal microphone, used to construct the error signal in the scheme, cannot be located exactly at the eardrum. Besides, the spatial distribution of sound sources and the geometry of the ear canal do not allow to achieve control over the complete ear canal. Therefore, the actual SNR at the eardrum is basically unknown and uncontrolled.

ANC allows to generate a zone-of-quiet based on destructive interference. The size and shape of this zone-of-quiet depend on the type of the sound sources and the frequency components of the signal to be cancelled [13][14][15]. It is therefore possible to determine the performance of the scheme described in [12] at any point in the ear canal, based on the spatial distribution of the sound sources and on the position of the ear canal microphone.

Capitalising on this idea, it is possible to define a particular design criterion, e.g., a mean squared error (MSE) criterion, at one particular point of the ear canal, away from the ear canal microphone, and to derive a filter from the minimisation of this MSE criterion to control the noise at this particular remote point (RP). The RP-based filter then exhibit a structure similar to the filtered-X structure.

In a similar way, it is also possible to define an average of 
the MSE criterion over a desired zone-of-quiet. A filter is then derived from the minimisation of this averaged-MSE (aMSE) criterion in order to achieve a control that is more robust on the desired zone-of-quiet compared to a scheme minimising a standard MSE criterion as in [12].

In [16] the authors presented an integrated ANC and NR scheme adjusted to a specific zone-of-quiet. This paper will first introduce a scheme that aims to minimise the noise at a RP and extend this scheme to a scheme designed to minimise the noise power over a desired zone-of-quiet. These two approaches are then applied to a ANC scheme and to the integrated ANC and NR scheme. The performance of these schemes will then be compared with the performance of the standard ANC scheme and the integrated ANC and NR scheme [12]. Instead of a time-domain approach as in [12], the frequency-domain approach presented in [17] is adopted here.

The signal model, the standard ANC scheme and integrated ANC and NR scheme are described in Section II. The ANC scheme and integrated ANC and NR scheme designed to control the noise at a RP are presented in Section III. The zone-of-quiet based approach to ANC and integrated ANC and NR, to control the noise over a desired zone, is presented in Section IV. Experimental results are presented in Section V and finally conclusions are presented in Section VI.

\section{BACKGROUND AND PROBLEM STATEMENT}

State-of-the-art speech enhancement in hearing aids is based on standard NR techniques ignoring the effects of noise leakage through the fitting and the secondary path between the loudspeaker and the eardrum. It has been shown in [12] that the leakage signal can significantly degrade the NR performance for hearing aid gains up to $20 \mathrm{~dB}$. It is possible however to attenuate the leakage signal's noise component using ANC. This section introduces the signal model and notations, suggests a frequency-domain multichannel ANC and introduces a frequency-domain version of the integrated ANC and NR scheme presented in [12].

\section{A. Signal model}

Let $M$ be the number of hearing aid microphones (channels). The frequency-domain signal $X_{m}$ for microphone $m$ has a desired speech part $X_{m}^{s}$ and an additive noise part $X_{m}^{n}$, i.e.:

$$
X_{m}(\omega)=X_{m}^{s}(\omega)+X_{m}^{n}(\omega) \quad m \in\{1 \ldots M\}
$$

where $\omega=2 \pi f$ is a frequency-domain variable. For conciseness, $\omega$ will be omitted in all subsequent equations.

In practice the frequency-domain signal $X_{m}$ is obtained by taking the discrete-time Fourier transform (DTFT) of the timedomain signal $x_{m}[k]$. Where $k$ is the time index.

In the sequel, superscripts $s$ and $n$ will also be used for other signals and vectors, to denote their speech and noise component, respectively. Signal model (1) holds for so-called "speech plus noise periods". There are also "noise only periods" (i.e. speech pauses), during which only a noise component is observed.
In practice, in order to distinguish "speech plus noise periods" from "noise only periods" it is necessary to use a voice activity detector (VAD). The performance of the VAD can affect the performance of the filters. In this paper however, as research on VAD is a vast subject in itself, a perfect VAD is assumed so as to focus on the performance improvement owing to the zone-of-quiet approach.

The compound vector gathering all microphone signals is:

$$
\mathbf{X}=\left[X_{1} \ldots X_{M}\right]^{T}
$$

A MWF $\mathbf{W}=\left[W_{1} \ldots W_{M}\right]^{T}$ will be designed and applied to these signals, which minimises an MSE criterion:

$$
J_{\mathrm{MSE}}=\mathbb{E}\left\{|E|^{2}\right\}
$$

Where $\mathbb{E}$ is the expectation operator and $E$ is an error signal to be defined next, depending on the scheme applied.

The filter output signal $Z$ (i.e., the signal to be fed into the hearing aid loudspeaker) is defined as:

$$
Z=\mathbf{W}^{H} \mathbf{X}
$$

where ${ }^{H}$ denotes the Hermitian transpose.

The desired speech signal, as defined in [12], is arbitrarily chosen to be the (unknown) speech component of the first microphone signal $(m=1)$, up to a delay $\Delta$. This can be written as:

$$
\begin{aligned}
D_{\mathrm{NR}} & =\mathbf{G}_{1, \Delta}^{H} \mathbf{X}^{s} \\
\mathbf{G}_{1, \Delta} & =\left[G e^{-j \omega \Delta} \mid 0 \ldots 0\right]=G \cdot \mathbf{e}_{1, \Delta}
\end{aligned}
$$

where the gain $G$ is the amplification that compensates for the hearing loss.

The autocorrelation matrices of the speech component and the noise component of the microphone signals are respectively given by:

$$
\begin{aligned}
& \mathbf{R}_{X^{s}}=\mathbb{E}\left\{\mathbf{X}^{s} \mathbf{X}^{s H}\right\} \\
& \mathbf{R}_{X^{n}}=\mathbb{E}\left\{\mathbf{X}^{n} \mathbf{X}^{n H}\right\}
\end{aligned}
$$

In a stationary scenario, and if the speech signal and the noise signal are assumed to be uncorrelated, $\mathbf{R}_{X^{n}}$ can be estimated during "noise only periods" and $\mathbf{R}_{X^{s}}$ can be estimated during "speech plus noise periods" using:

$$
\begin{aligned}
\mathbf{R}_{X} & =\mathbb{E}\left\{\mathbf{X} \mathbf{X}^{H}\right\} \\
\mathbf{R}_{X^{s}} & =\mathbf{R}_{X}-\mathbf{R}_{X^{n}}
\end{aligned}
$$

In practice, the correlation matrices are estimated recursively. The estimate of the autocorrelation matrix of the microphone signals is updated during "speech plus noise periods", using:

$$
\tilde{\mathbf{R}}_{X}=\lambda \tilde{\mathbf{R}}_{X}+(1-\lambda) \mathbf{X} \mathbf{X}^{H}
$$

where $\lambda \in[0,1]$ is an exponential forgetting factor that depends on the number of past samples to be taken into account. Here $\lambda=1-\frac{1}{N_{\text {samples }}}$ with $N_{\text {samples }}=20000$ samples at $16 \mathrm{kHz}$. This clearly exceeds the spectral stationarity of speech signals (around 20ms) but not necessarily the spatial stationarity of the sources. It also allows to have a stable estimation for the correlation matrices, which is the main concern at this stage. 
The estimate of the autocorrelation matrix of the noise component of the microphone signals is updated similarly during "noise only periods", using:

$$
\begin{aligned}
\tilde{\mathbf{R}}_{X^{n}} & =\lambda \tilde{\mathbf{R}}_{X^{n}}+(1-\lambda) \mathbf{X} \mathbf{X}^{H} \\
& =\lambda \tilde{\mathbf{R}}_{X^{n}}+(1-\lambda) \mathbf{X}^{n} \mathbf{X}^{n H}
\end{aligned}
$$

If speech and noise are uncorrelated, the estimate of the autocorrelation matrix of the speech component of the microphone signal is then given by:

$$
\tilde{\mathbf{R}}_{X^{s}}=\tilde{\mathbf{R}}_{X}-\tilde{\mathbf{R}}_{X^{n}}
$$

\section{B. MWF-based noise reduction, secondary path and signal leakage}

The MWF-based NR is designed to minimise the squared distance between the filtered microphone signal $\left(\mathbf{W}^{H} \mathbf{X}\right)$ and the desired speech signal $\left(D_{\mathrm{NR}}=\mathbf{G}_{1, \Delta} \mathbf{X}^{s}\right)$. Therefore, the MSE criterion to be minimised is:

$$
J_{\mathrm{NR}}=\mathbb{E}\left\{\left|\mathbf{W}^{H} \mathbf{X}-\mathbf{G}_{1, \Delta}^{H} \mathbf{X}^{s}\right|^{2}\right\}
$$

If speech and noise are uncorrelated, the corresponding Wiener filter is:

$$
\mathbf{W}_{\mathrm{NR}}=\mathbf{R}_{X}^{-1} \mathbf{R}_{X^{s}} \mathbf{G}_{1, \Delta}
$$

The filter (16) is designed without taking the effects of the signal leakage and the secondary path effects into account. Figure (1) shows an MWF-based NR where the secondary path and the signal leakage are added.

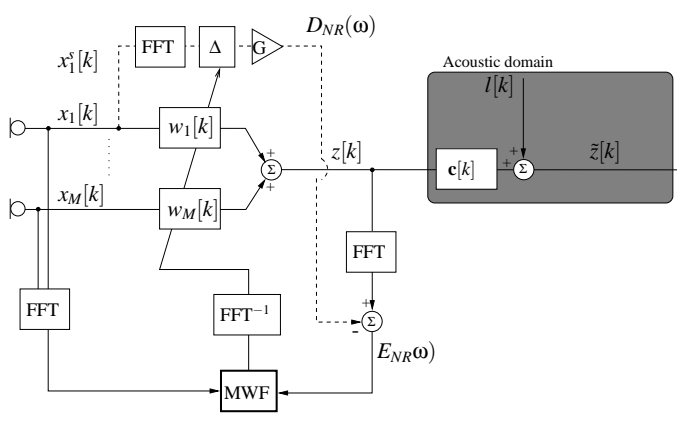

Fig. 1. MWF-based NR

The secondary path represents the propagation from the loudspeaker to the eardrum. In the case of hearing aids with an open fitting, this secondary path includes the effects of the transfer function that models the acoustic propagation from the loudspeaker to the eardrum (including the loudspeaker response itself) and the effects of the sound transferred from the ear canal to the open field through the open fitting (also know as vent-loss). In the context of hearing aids, this secondary path then usually acts as an attenuation. Assuming that the loudspeaker characteristic is approximately linear, the secondary path can be represented by a filter coefficient vector $\mathbf{c}[k]$ of length $P$. The DFT of $\mathbf{c}[k]$ is then denoted $C$.

A hearing aid with an open fitting has no earmold to prevent ambient sound from leaking into the ear canal, which results in an additional leakage signal $L$ reaching the eardrum. In literature this leakage signal is also referred to as vent-through or direct sound [18][19]. No direct processing can be done on this signal, therefore its SNR is generally lower than for the signal provided by the hearing aid.

Taking both the leakage signal and the secondary path effect into account, leads to the following output signal model:

$$
\tilde{Z}=C \cdot Z+L
$$

It clearly appears that for small amplification gains $G$ the leakage SNR may affect the output SNR thus partly cancelling the improvement achieved with the NR. In conclusion, whereas the secondary path and the leakage are not taken into account in conventional NR algorithms, they may degrade their performance significantly.

\section{Active noise control}

The leakage signal is not processed in the hearing aid therefore it is not possible to improve its SNR using standard NR algorithms. It is possible however to attenuate the leakage signal using ANC. In all subsequent systems, it is assumed that a microphone is present in the ear canal to provide an error signal which will be used by the ANC. This subsection presents a frequency-domain multichannel ANC used to attenuate the leakage signal $L$ as shown in Figure (3).

The performance of a feedforward ANC schemes is highly dependent on the causality of the system [7]. The distance between the reference microphones and the secondary source must be sufficient to allow causal design. In the case of hearing aids with an open fitting, the causality criterion (Figure 2) can be defined as follows:

The acoustic delay from the noise source to the ear canal microphone $\Delta_{\text {pri }}$ has to be larger than the sum of the acoustic delay from the source to one of the reference microphones $\Delta_{\text {ref }}$, the delay associated with the processing within the hearing aid $\Delta_{\mathrm{HA}}$ (i.e., Analog-to-Digital converter delays, Digital-toAnalog converter delays...), the algorithmic delay $\Delta_{\text {alg }}$ (delay introduced by, e.g., NR, ANC, feedback control...), and the acoustic delay of the secondary path $\Delta_{\text {sec }}$. The acoustic delay is the direct propagation time between a sound source and a microphone (BTE microphone or ear canal microphone). If the source is about 1 meter away from the listener, $\Delta_{\text {pri }}$ and $\Delta_{\text {ref }}$ are about 50 taps (i.e., sampling periods) at $16 \mathrm{kHz}$ (with $\Delta_{\text {pri }} \geq \Delta_{\text {ref }}$ ). In hearing aids, $\Delta_{\text {sec }}$ is just a few taps at $16 k H z$.

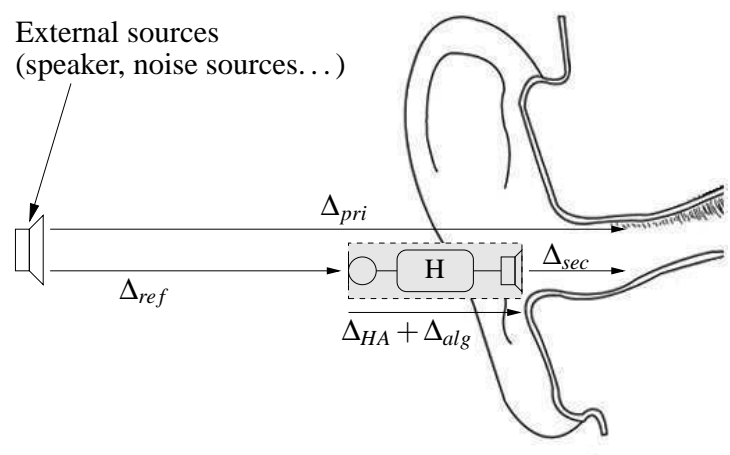

Fig. 2. Delays in hearing aid system 


$$
\Delta_{\text {ref }}+\Delta_{\mathrm{HA}}+\Delta_{\text {alg }}+\Delta_{\text {sec }} \leq \Delta_{\text {pri }}
$$

The causality margin $\delta$ is then defined as:

$$
\delta=\Delta_{\text {pri }}-\left(\Delta_{\text {ref }}+\Delta_{\text {HA }}+\Delta_{\text {sec }}\right)
$$

, i.e., the delay (number of taps) that can be introduced by the DSP algorithms in the hearing aids such that the system still satisfies (18):

$$
\Delta_{\text {alg }} \leq \delta
$$

When $\delta \geq 0$ the DSP algorithms can introduce a delay $\Delta_{\text {alg }} \geq 0$. It is then possible to design a causal ANC. When $\delta<0$ the ANC has to be designed as a non-causal filter.

In practice, this criterion does not define a hard limit but it gives an indication on the performance to be expected from an ANC scheme. The bandwidth on which it is possible to achieve good ANC performance reduces with the causality margin $\delta$ specified in (19). When (20) is not satisfied, the ANC efficiency vanishes quickly [20]. Delay is thus a critical problem in ANC and many approaches have been developed to try to deal with it [21], [22].

In practice, to ensure the causality of the scheme, the filter coefficients are computed in the frequency-domain while the actual filtering operation is performed in the time-domain, in a similar way as presented in [21]. The time-domain delayless ANC filter is obtained by taking the $2 N$-IDFT of the frequency-domain vector coefficient. The resulting timedomain filter contains an $N$-dimensional causal part and a $N$ dimensional anticausal part. The time-domain filter effectively applied to the microphone signals is truncated to the $\mathrm{N}$ dimensional causal part. The algorithmic delay introduced by the filter then reduces to $\Delta_{\text {alg }}=1$.

Note that, due to the inverse DFT and the truncation, the effect of causality on the frequency-domain version of the ANC schemes is unclear and difficult to analyse. Therefore, in this article the frequency-domain ANC schemes are mainly operating on a system with a sufficiently positive causality margin $(\delta \geq 0)$, so that the effect of the truncation is indeed limited. This corresponds to neglecting the hearing aid delays $\left(\Delta_{\mathrm{HA}}=0\right)$ in which case the causality margin typically varies between 0 taps and 3 taps (at $16 \mathrm{kHz}$ ), depending on the geometry of the sources. A study of the impact of causality on the performance of the time-domain integrated ANC and NR scheme can be found in [12].

In this paper, the DFT is applied with a $2 N-1$-tap overlap in order to obtain a filter as close as possible to the optimal filter at each time instant $k$. This overlap value leads to a high complexity algorithm. In practice, the system to identify is varying slowly and the signals can be considered to be stationnary over at least 20 to $30 \mathrm{~ms}$. The overlap could then easily be reduced to $N$. With this overlap value and using an MWF implementation based, e.g., on general singular value decomposition [23], it could be realistic to consider implementing the FxMWF in a real-time system.

All subsequent schemes rely on a filtered-X type operation based on an estimate $\hat{C}$ of the secondary path $C$. The filtered

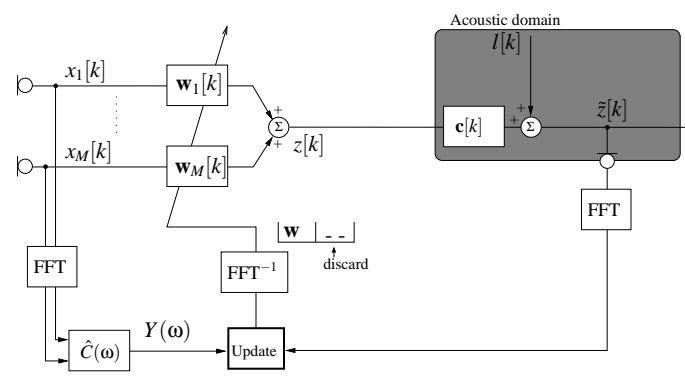

Fig. 3. Multichannel ANC

microphone signals are then:

$$
\begin{aligned}
Y_{m} & =\hat{C} \cdot X_{m} \quad m \in\{1 \ldots M\} \\
\mathbf{Y}^{T} & =\left[Y_{1} \ldots Y_{M}\right]
\end{aligned}
$$

The secondary path can be estimated off-line using classic identification methods based for example on Least Mean Square (LMS) algorithms [24], or on-line by adding random noise to the signal exciting the secondary path, as introduced by Eriksson et al. in [25] and later refined by Kuo et al. [26] and Zhang et al. [27].

The ANC output signal is $\mathbf{W}^{H} \mathbf{X}$, where the filter $\mathbf{W}$ is designed to minimise the MSE:

$$
J_{\mathrm{ANC}}=\mathbb{E}\left\{\left|C \cdot \mathbf{W}^{H} \mathbf{X}+L\right|^{2}\right\}
$$

Assuming that the secondary path identification error is small $(\hat{C} \approx C)$ and that the filter $\mathbf{W}$ is adapting slowly, the MSE criterion (22) can be written as follows:

$$
J_{\mathrm{ANC}} \approx \mathbb{E}\left\{\left|\mathbf{W}^{H} \mathbf{Y}+L\right|^{2}\right\}
$$

The FxMWF minimising (22) is then:

$$
\mathbf{W}_{\mathrm{ANC}}=-\mathbf{R}_{Y}^{-1} \mathbf{r}_{Y L}
$$

Here $\mathbf{R}_{Y}$ is the correlation matrix of the filtered microphone signals $\mathbf{Y}$ and $\mathbf{r}_{Y L}$ is the cross-correlation vector between the filtered microphone signals $\mathbf{Y}$ and the leakage signal $L$.

$$
\begin{aligned}
\mathbf{R}_{Y} & =\mathbb{E}\left\{\mathbf{Y} \mathbf{Y}^{H}\right\} \\
\mathbf{r}_{Y L} & =\mathbb{E}\left\{\mathbf{Y} L^{*}\right\}
\end{aligned}
$$

where ${ }^{*}$ denotes the conjugate of a complex number. The leakage signal $L$ can be estimated from the error signal $\tilde{Z}$ as follows:

$$
L=\tilde{Z}-C \cdot \mathbf{W}^{H} \mathbf{X} \approx \tilde{Z}-C \cdot Y
$$

In practice the cross-correlation vector is then estimated recursively using:

$$
\tilde{\mathbf{r}}_{Y L}=\lambda \tilde{\mathbf{r}}_{Y L}+(1-\lambda) \mathbf{Y} L^{*}
$$

\section{Integrated active noise control and noise reduction}

Filter (24) can be used to compensate for the signal leakage. In the speech enhancement context however, the goal is to deliver a desired speech signal at the user's eardrum. The ANC then has to be performed together with the NR.

This subsection presents a frequency-domain version of the scheme introduced in [12] which integrates the ANC and the NR in a single set of adaptive filters and which is shown in 


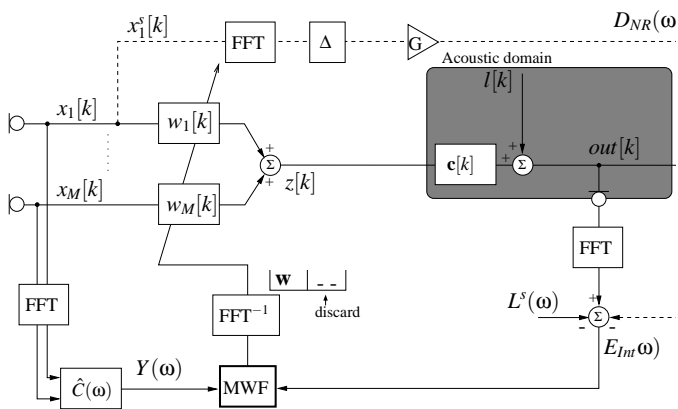

Fig. 4. Integrated ANC and NR

Figure (4). The purpose of the NR is to provide an optimal estimate of the desired speech signal $D_{\mathrm{NR}}(5)$. The aim is to deliver this desired speech signal at the eardrum in spite of secondary path effects and the signal leakage.

The purpose of the ANC is now to cancel the noise component $L^{n}$ of the leakage signal $L$ arriving at the eardrum. In the hearing aids context, the speech component of the leakage signal can provide cues which, e.g., are helpful for speaker localisation. Therefore, it is chosen here to cancel only the noise component of the leakage signal and preserve its speech component.

The overall desired signal (at the eardrum) to be used is then:

$$
D_{\mathrm{Int}}=D_{\mathrm{NR}}+L^{s}
$$

Hence the MSE criterion to be minimised is:

$$
J_{\text {Int }}=\mathbb{E}\left\{\left|C \cdot \mathbf{W}^{H} \mathbf{X}+L^{n}-D_{\mathrm{NR}}\right|^{2}\right\}
$$

Assuming that the secondary path identification error is small $(\hat{C} \approx C)$ and that the filter $\mathbf{W}$ is adapting slowly, the MSE criterion (30) can be written as follows:

$$
J_{\text {Int }} \approx \mathbb{E}\left\{\left|\mathbf{W}^{H} \mathbf{Y}+L^{n}-D_{\mathrm{NR}}\right|^{2}\right\}
$$

The FxMWF minimising (30) is then:

$$
\mathbf{W}_{\mathrm{Int}}=\mathbf{R}_{Y}^{-1}\left(\mathbf{r}_{Y D_{\mathrm{NR}}}-\mathbf{r}_{Y L^{n}}\right)=\mathbf{R}_{Y}^{-1}\left(\mathbf{r}_{Y^{s} D_{\mathrm{NR}}}-\mathbf{r}_{Y^{n} L^{n}}\right)
$$

Here $\mathbf{r}_{Y^{s} D_{\mathrm{NR}}}$ and $\mathbf{r}_{Y^{n} L^{n}}$ are the cross-correlation vectors between the filtered microphone signals $\mathbf{Y}$ and the desired signal $D_{\mathrm{NR}}$ and the noise component of the leakage signal $L^{n}$, respectively.

$$
\begin{aligned}
\mathbf{r}_{Y^{s} D_{\mathrm{NR}}} & =\mathbb{E}\left\{\mathbf{Y} D_{\mathrm{NR}}^{*}\right\}=\mathbf{G}_{1, \Delta}^{H} \mathbb{E}\left\{\mathbf{Y}^{s} \mathbf{X}^{s H}\right\} \\
\mathbf{r}_{Y^{n} L^{n}} & =\mathbb{E}\left\{\mathbf{Y}^{n} L^{n *}\right\}
\end{aligned}
$$

Where the noise component of the leakage signal $L$ can be estimated, during "noise only periods", from the error signal $\tilde{Z}^{n}$ as follows:

$$
L^{n}=\tilde{Z}^{n}-C \cdot \mathbf{W}^{H} \mathbf{X}^{n} \approx \tilde{Z}^{n}-C \cdot Y^{n}
$$

In practice, the cross-correlation vectors are then estimated recursively according to (28).

The time-domain version of the above scheme, based on an adaptation employing an error signal (30) measured with the ear canal microphone, has been derived and evaluated in [12].

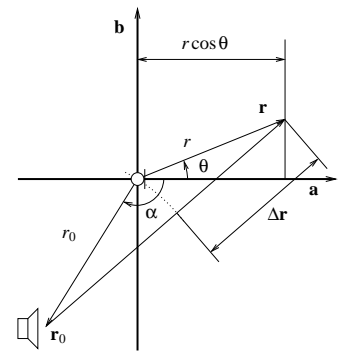

(a) Geometric description

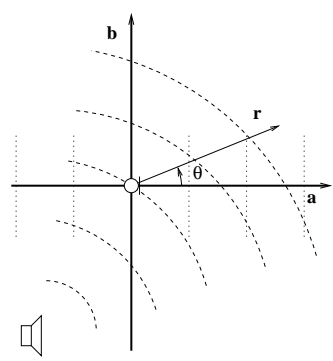

(b) Sound field
Fig. 5. Sources distribution and sound field. The dashed lines represent the sound field from the secondary source. The dotted lines represent the sound field from the leakage.

\section{INTEGRATED ACTIVE NOISE CONTROL AND NOISE REDUCTION AT A REMOTE POINT}

The scheme described above achieves noise control at the ear canal microphone. Ideally, this should be located at the eardrum. In practice, however, this is not possible, i.e., the controlled point can be only located a few millimetres away from the eardrum. The ANC can then only generate a socalled zone-of-quiet around the ear canal microphone and the signal which is actually reaching the eardrum is unknown and uncontrolled.

\section{A. Remote point model}

To study the performance of the ANC and NR schemes over the zone-of-quiet, the ear canal microphone is chosen to be the origin of the 2-dimensional coordinate system used to determine each point of the space (presented in Figure 5(a)). It is then possible to estimate the sound pressure at a particular point $\mathbf{r}=(r \sin \theta, r \cos \theta)$ in the ear canal, when the sound pressure at the ear canal microphone and the type of the sound sources generating the sound field in the ear canal are known [13][14][15].

The external sound sources (the speech source and the noise sources) are assumed to be far enough from the hearing aid user so that the leakage signal can be viewed as a far field signal and hence a plane wave model can be used. Assuming also that the ear canal acts as a waveguide and that the plane waves from different directions are uncorrelated [28], the sound field generated by the leakage can be modelled as one plane wave propagating along the ear canal axis (chosen to be the axis a) as presented on Figure 5(b). The sound pressure at point $\mathbf{r}$ resulting from the leakage signal can then be expressed as follows:

$$
L(\mathbf{r})=L(\mathbf{0}) \delta_{1}(\mathbf{r})
$$

where

$$
\delta_{1}(\mathbf{r}) \triangleq \mathrm{e}^{-j \omega \frac{r \cos \theta}{c_{0}}}
$$

with $c_{0}$ the speed of sound in air and $L(\mathbf{0})$ the sound pressure resulting from the leakage signal at the ear canal microphone.

The hearing aid loudspeaker (secondary source), is located at $\mathbf{r}_{0}=\left(r_{0} \sin \alpha, r_{0} \cos \alpha\right)$. The ear canal is assumed to be in the near field of the hearing aid loudspeaker. A simple model to study the sound field generated by the hearing aid loudspeaker is then the monopole-point-secondary-source model [14]. The use of this model can be justified by the small dimension of the hearing aid loudspeaker. The sound 
field generated by the hearing aid loudspeaker is then assumed to be circular with decaying amplitude:

$$
U(\mathbf{r})=U(\mathbf{0}) \delta_{2}(\mathbf{r})
$$

where

$$
\delta_{2}(\mathbf{r}) \triangleq \frac{r_{0}}{\left|\mathbf{r}-\mathbf{r}_{0}\right|} \mathrm{e}^{-j \omega \frac{\Delta \mathbf{r}}{c_{0}}}
$$

with $U(\mathbf{0})$ the sound pressure resulting from the hearing aid signal $Z$ at the ear canal microphone

$$
U(\mathbf{0})=C \cdot Z
$$

and $\Delta r$ defined in Figure (5(a)).

Note that the subsequent schemes can be adapted if these acoustic models are replaced by other models. The methodology applied stays valid as long as it is possible to find a mathematical model to describe the acoustic propagation from the sources (speech source, noise sources and secondary source) to the eardrum.

Using Parseval's theorem, the power of the total sound pressure signal and the SNR at point $\mathbf{r}$ can be expressed as follows:

$$
\begin{aligned}
\operatorname{Pow}(\mathbf{r}) & =\frac{1}{2 \pi} \int_{-\pi}^{\pi}\left|\delta_{1}(\mathbf{r}) L(\mathbf{0})+\delta_{2}(\mathbf{r}) U(\mathbf{0})\right|^{2} \mathrm{~d} \omega \\
\operatorname{SNR}(\mathbf{r}) & =\frac{\operatorname{Pow}^{s}(\mathbf{r})}{\operatorname{Pow}^{n}(\mathbf{r})}
\end{aligned}
$$

Interferences which are destructive at the ear canal microphone (thus allowing ANC) may become constructive at a RP. The noise component of the leakage may then be amplified at the eardrum instead of being attenuated, resulting in a low $\operatorname{SNR}(\mathbf{r})$.

\section{B. Active noise control}

When the noise is to be controlled at a point $\mathbf{r}$ away from the ear canal microphone and when the acoustic propagation from the sources to the RP can be modelled, the MSE criterion (22) can be rewritten at point $\mathbf{r}$ based on equations (38) and (36):

$$
J_{\mathrm{ANC}}(\mathbf{r})=\mathbb{E}\left\{\left|\delta_{2}(\mathbf{r}) C \mathbf{W}^{H} \mathbf{X}+\delta_{1}(\mathbf{r}) L\right|^{2}\right\}
$$

Assuming that the secondary path identification error is small $(\hat{C} \approx C)$ and that the filter $\mathbf{W}$ is adapting slowly, the MSE criterion (43) can be written as follows:

$$
J_{\mathrm{ANC}}(\mathbf{r}) \approx \mathbb{E}\left\{\left|\delta_{2}(\mathbf{r}) \mathbf{W}^{H} \mathbf{Y}+\delta_{1}(\mathbf{r}) L\right|^{2}\right\}
$$

The FxMWF minimising (43) is then:

$$
\mathbf{W}_{\mathrm{ANC}}(\mathbf{r})=-\frac{\delta_{2}(\mathbf{r}) \delta_{1}(\mathbf{r})^{*}}{\left|\delta_{2}(\mathbf{r})\right|^{2}} \mathbf{R}_{Y}^{-1} \mathbf{r}_{Y L}
$$

Alternatively, (44) can be written as follows:

$$
J_{\mathrm{ANC}}(\mathbf{r}) \approx \mathbb{E}\left\{\left|\mathbf{W}^{H} \mathbf{Y}(\mathbf{r})+L(\mathbf{r})\right|^{2}\right\}
$$

and then filter (45) reduces to:

$$
\mathbf{W}_{\mathrm{ANC}}(\mathbf{r})=-\mathbf{R}_{Y}(\mathbf{r})^{-1} \mathbf{r}_{Y L}(\mathbf{r})
$$

Here $\mathbf{R}_{Y}(\mathbf{r})$ is the correlation matrix of $\mathbf{Y}(\mathbf{r})$, i.e., the filtered microphone signals $\mathbf{Y}$ estimated at the RP $\mathbf{r}$ and $\mathbf{r}_{Y L}(\mathbf{r})$ is the cross-correlation vector between $\mathbf{Y}(\mathbf{r})$ and $L(\mathbf{r})$, i.e., the leakage signal $L$ at the RP $\mathbf{r}$.

$$
\begin{aligned}
\mathbf{R}_{Y}(\mathbf{r}) & =\mathbb{E}\left\{\mathbf{Y}(\mathbf{r}) \mathbf{Y}(\mathbf{r})^{H}\right\} \\
\mathbf{r}_{Y L}(\mathbf{r}) & =\mathbb{E}\left\{\mathbf{Y}(\mathbf{r}) L(\mathbf{r})^{*}\right\}
\end{aligned}
$$

Filter (47) is then a FxMWF-based ANC filter applied on the filtered microphone signals $Y$ to attenuate the leakage signal $L$ both filtered by the corresponding acoustic models $\delta_{2}(\mathbf{r})$ and $\delta_{1}(\mathbf{r})$, respectively. It can then be used to compensate for the signal leakage at a distant point $\mathbf{r}$.

The cost function (46) can be rewritten as follows:

$$
J_{\mathrm{ANC}}(\mathbf{r}) \approx \mathbb{E}\left\{\left|C(\mathbf{r}) \mathbf{W}^{H} \mathbf{X}+L(\mathbf{r})\right|^{2}\right\}
$$

The RP-based ANC scheme is then merely an FxMWFbased ANC canceling the noise $L(\mathbf{r})$, where the secondary path $C$ is replaced by $C(\mathbf{r})=\delta_{2}(\mathbf{r}) C$.

\section{Integrated active noise control and noise reduction}

In a similar way, a filter $\mathbf{W}_{\text {Int }}(\mathbf{r})$ may be defined corresponding to a RP r. This filter then minimises the MSE criterion (30) rewritten at point $\mathbf{r}$, based on (36) and (38):

$$
J_{\text {Int }}(\mathbf{r})=\mathbb{E}\left\{\left|\delta_{2}(\mathbf{r}) C \mathbf{W}^{H} \mathbf{X}+\delta_{1}(\mathbf{r}) L^{n}-D_{\mathrm{NR}}\right|^{2}\right\}
$$

Assuming that the secondary path identification error is small $(\hat{C} \approx C)$ and that the filter $\mathbf{W}$ is adapting slowly, the MSE criterion (51) can be written as follows:

$$
J_{\text {Int }}(\mathbf{r}) \approx \mathbb{E}\left\{\left|\delta_{2}(\mathbf{r}) \mathbf{W}^{H} \mathbf{Y}+\delta_{1}(\mathbf{r}) L^{n}-D_{\mathrm{NR}}\right|^{2}\right\}
$$

Assuming that the speech and noise component of the signals are uncorrelated, the optimal filter (FxMWF) minimising (52) is then:

$$
\begin{aligned}
& \mathbf{W}_{\text {Int }}(\mathbf{r})=\frac{\mathbf{R}_{Y}^{-1}\left(\delta_{2}(\mathbf{r}) \mathbf{r}_{Y D_{\mathrm{NR}}}-\delta_{2}(\mathbf{r}) \delta_{1}(\mathbf{r})^{*} \mathbf{r}_{Y L^{n}}\right)}{\left|\delta_{2}(\mathbf{r})\right|^{2}} \\
& \mathbf{W}_{\text {Int }}(\mathbf{r})=\frac{\mathbf{R}_{Y}^{-1}\left(\delta_{2}(\mathbf{r}) \mathbf{r}_{Y^{s} D_{\mathrm{NR}}}-\delta_{2}(\mathbf{r}) \delta_{1}(\mathbf{r})^{*} \mathbf{r}_{Y^{n} L^{n}}\right)}{\left|\delta_{2}(\mathbf{r})\right|^{2}}
\end{aligned}
$$

Filter (53) then allows to deliver a desired speech signal and compensate for the noise component of the leakage signal at a point $\mathbf{r}$ which is away from the ear canal microphone.

Alternatively, (52) can be written as:

$$
J_{\mathrm{Int}}(\mathbf{r}) \approx \mathbb{E}\left\{\left|\mathbf{W}^{H} \mathbf{Y}(\mathbf{r})+L^{n}(\mathbf{r})-D_{\mathrm{NR}}\right|^{2}\right\}
$$

and then filter $(45)$ reduces to:

$$
\mathbf{W}_{\text {Int }}(\mathbf{r})=\mathbf{R}_{Y}(\mathbf{r})^{-1} \mathbf{r}_{Y D_{\text {Int }}}(\mathbf{r})
$$

Here $\mathbf{r}_{Y D_{\text {Int }}}(\mathbf{r})$ is the cross-correlation vector between the filtered microphone signals $\mathbf{Y}$ and the desired signal $D_{\text {Int }}$ at the RP r.

$$
\mathbf{r}_{Y D_{\text {Int }}}(\mathbf{r})=\mathbb{E}\left\{\mathbf{Y}(\mathbf{r}) D_{\text {Int }}(\mathbf{r})^{*}\right\}
$$

Assuming that speech and noise are uncorrelated $\mathbf{r}_{Y D_{\mathrm{Int}}}(\mathbf{r})$ can be expressed as follows:

$$
\begin{aligned}
\mathbf{r}_{Y D_{\mathrm{Int}}}(\mathbf{r}) & =\mathbf{r}_{Y^{s} D_{\mathrm{NR}}}(\mathbf{r})-\mathbf{r}_{Y^{n} L^{n}}(\mathbf{r}) \\
& =\mathbb{E}\left\{\mathbf{Y}^{s}(\mathbf{r}) D_{\mathrm{NR}}(\mathbf{r})^{*}\right\}-\mathbb{E}\left\{\mathbf{Y}^{n}(\mathbf{r}) L^{n}(\mathbf{r})^{*}\right\}
\end{aligned}
$$


Filter (55) can then be rewritten as:

$$
\mathbf{W}_{\text {Int }}(\mathbf{r})=\mathbf{R}_{Y}(\mathbf{r})^{-1}\left(\mathbf{r}_{Y^{s} D_{\mathrm{NR}}}(\mathbf{r})-\mathbf{r}_{Y^{n} L^{n}}(\mathbf{r})\right)
$$

Filter (58) provides an integrated ANC and NR applied to the filtered microphone signals $\mathbf{Y}$ and the leakage signal $L$ both estimated at the RP r.

The cost function (54) can be rewritten as follows:

$$
J_{\mathrm{MSE}}(\mathbf{r}) \approx \mathbb{E}\left\{\left|C(\mathbf{r}) \mathbf{W}^{H} \mathbf{X}+L^{n}(\mathbf{r})-D_{\mathrm{NR}}\right|^{2}\right\}
$$

The RP-based integrated ANC and NR scheme is then merely and FxMWF-based integrated ANC and NR cancelling the noise $L^{n}(\mathbf{r})$ and delivering the desired speech signal $D_{\mathrm{NR}}$, where the secondary path $C$ is replaced by $C(\mathbf{r})$.

\section{Single speech source scenario}

It has been explained in [17] that the leakage signal can be approximated by a linear combination of the input signals

$$
L=\tilde{\mathbf{P}}^{H} \mathbf{X}+e_{L}
$$

The leakage signal at point $\mathbf{r}$ can similarly be approximated by a linear combination of the inputs:

$$
L(\mathbf{r})=\delta_{1}(\mathbf{r}) L(\mathbf{0})=\tilde{\mathbf{P}}^{H}(\mathbf{r}) \mathbf{X}+e_{L}(\mathbf{r})
$$

where

$$
\begin{aligned}
\tilde{\mathbf{P}}(\mathbf{r}) & =\delta_{1}(\mathbf{r}) \tilde{\mathbf{P}} \\
e_{L}(\mathbf{r}) & =\delta_{1}(\mathbf{r}) e_{L}
\end{aligned}
$$

The estimation error $e_{L}(\mathbf{r})$ is then orthogonal to the microphone signals and to the microphone signals filtered by $\tilde{\mathbf{P}} \tilde{\mathbf{r}})$ [24]. If the filter $\mathbf{W}(\mathbf{r})$ varies slowly $e_{L}(\mathbf{r})$ is also orthogonal to the microphone signals filtered by $\mathbf{W}$.

$$
\begin{aligned}
\mathbb{E}\left\{\mathbf{X}(\omega) e_{L}(\omega)^{*}\right\} & =0 \\
\mathbb{E}\left\{\tilde{\mathbf{P}}(\omega)^{H} \mathbf{X} e_{L}(\omega)^{*}\right\} & =0 \\
\mathbb{E}\left\{\mathbf{W}(\omega)^{H} \mathbf{X}(\omega) e_{L}(\omega)^{*}\right\} & =0
\end{aligned}
$$

The integrated ANC and NR scheme at RP r (55) can then be rewritten as:

$\mathbf{W}_{\text {Int }}(\mathbf{r})=\frac{C^{*}}{\left|\delta_{2}(\mathbf{r}) C\right|^{2}} \mathbf{R}_{X}^{-1}\left(\delta_{2}(\mathbf{r}) \mathbf{R}_{X^{s}} \mathbf{G}_{1, D_{\mathrm{NR}}}-\delta_{2}(\mathbf{r}) \mathbf{R}_{x^{n}} \tilde{\mathbf{P}}(\mathbf{r})^{H}\right)$

The filter (67) then reduces to:

$$
\mathbf{W}_{\text {Int }}(\mathbf{r})=\frac{C(\mathbf{r})^{*}}{|C(\mathbf{r})|^{2}} \mathbf{R}_{X}^{-1}\left(\mathbf{R}_{X^{s}} \mathbf{G} 1, D_{\mathrm{NR}}-\mathbf{R}_{x^{n}} \tilde{\mathbf{P}}(\mathbf{r})^{H}\right)
$$

In a single speech source scenario, the autocorrelation matrix of the microphone signals is rank-1 and applying the Woodbury identity to invert $\mathbf{R}_{X}$ leads to:

$$
\mathbf{W}_{\text {Int }}(\mathbf{r})=\frac{C(\mathbf{r})^{*}}{|C(\mathbf{r})|^{2}}\left[\frac{\mathbf{R}_{X}^{-1} \mathbf{R}_{X^{s}}}{1+\rho}\left(\mathbf{G}_{1, D_{\mathrm{NR}}}+\tilde{\mathbf{P}}(\mathbf{r})\right)-\tilde{\mathbf{P}}(\mathbf{r})\right]
$$

The output SNR of the RP-based integrated ANC and NR scheme estimated at point (r) is given by

$$
S N R(\mathbf{r})=\frac{\mathbb{E}\left\{\left|C(\mathbf{r})^{*} \mathbf{W}^{H} \mathbf{X}^{s}+\mathbf{X}^{s} \tilde{\mathbf{P}}(\mathbf{r})^{H}\right|^{2}\right\}}{\mathbb{E}\left\{\left.\left|C(\mathbf{r})^{*} \mathbf{W}^{H} \mathbf{X}^{n}+\mathbf{X}^{n} \tilde{\mathbf{P}}(\mathbf{r})^{H}\right|^{2}\right|^{2}\right\}}
$$

Depending on the number of sound sources (speech source plus noise sources) the output SNR is then:

$$
\begin{aligned}
S N R_{\operatorname{Int}(Q \leq M)}= & \rho=S N R_{N R(\text { noLeakage })} \\
S N R_{\operatorname{Int}(Q>M)}= & \frac{\frac{\rho^{2}}{(\rho+1)^{2}}\left(P_{D_{N R}}+\beta+\tilde{\mathbf{P}}^{H} R_{s} \tilde{\mathbf{P}}\right)+E_{e_{L}^{s}}}{\frac{\rho}{(\rho+1)^{2}}\left(P_{D_{N R}}+\beta+\tilde{\mathbf{P}}^{H} R_{s} \tilde{\mathbf{P}}\right)+E_{e_{L}^{n}}}
\end{aligned}
$$

The remote-point integrated $\mathrm{ANC}$ and NR then allows to restore the performance of the integrated $\mathrm{ANC}$ and $\mathrm{NR}$ at point $\mathbf{r}$ [17].

\section{INTEGRATED ACTIVE NOISE CONTROL AND NOISE REDUCTION OVER A ZONE-OF-QUIET}

The ultimate goal is to control the signal at the eardrum. Therefore, it is more relevant to design a filter adapted to a desired zone-of-quiet rather than a single RP. Capitalizing on the RP-approach, this section introduces the Zone-ofQuiet (ZQ) model, the ZQ-based ANC scheme and the ZQbased integrated ANC and NR scheme derived based on this ZQ model. The latter scheme has previously been presented in [16].

\section{A. Zone-of-quiet model}

The average signal power and the average SNR over a desired zone-of-quiet $S$ are defined as:

$$
\begin{aligned}
& \operatorname{aPow}(S) \triangleq \frac{1}{\bar{S}} \int_{S} \operatorname{Pow}(\mathbf{r}) \mathrm{d} S \\
& \operatorname{aSNR}(S) \triangleq \frac{1}{\bar{S}} \int_{S} \operatorname{SNR}(\mathbf{r}) \mathrm{d} S
\end{aligned}
$$

where $\bar{S}$ is the area of $S$.

The filters introduced in the previous sections minimise MSE criteria at a particular point (the ear canal microphone in Section II or a RP in Section III) and may exhibit degraded performance in terms of average signal power or average SNR over a desired ZQ (see also Section V).

In order to derive new filters which are efficient on a desired ZQ $S$ the average MSE (aMSE) criterion over a desired ZQ has to be defined:

$$
a J_{\mathrm{MSE}}(S)=\frac{1}{\bar{S}} \int_{S} J_{\mathrm{MSE}}(\mathbf{r}) \mathrm{d} S
$$

\section{B. Active noise control}

When the noise is to be controlled over a desired ZQ $S$ away from the ear canal microphone and when the acoustic propagation from the sources to a RP can be modelled, the aMSE criterion to be minimised can be written as:

$$
\begin{aligned}
a J_{\mathrm{ANC}}(S) & =\frac{1}{\bar{S}} \int_{S} \mathbb{E}\left\{\left|E_{\mathrm{ANC}}(\mathbf{r})\right|^{2}\right\} \mathrm{d} S \\
E_{\mathrm{ANC}}(\mathbf{r}) & =C \cdot \mathbf{W}^{H} \mathbf{X}(\mathbf{r})+L(\mathbf{r})
\end{aligned}
$$

Assuming that the secondary path identification error is small $(\hat{C} \approx C$ ) and that the filter $\mathbf{W}$ is adapting slowly, the aMSE criterion (75) can be written as follows:

$$
a J_{\mathrm{ANC}}(S) \approx \frac{1}{\bar{S}} \int_{S} \mathbb{E}\left\{\left|\delta_{2}(\mathbf{r}) \mathbf{W}^{H} \mathbf{Y}+\delta_{1}(\mathbf{r}) L\right|^{2}\right\} \mathrm{d} S
$$


The integration being a linear operator, the aMSE criterion can be rewritten as:

$$
\begin{aligned}
a J_{\mathrm{ANC}}(S) \approx & \frac{\mathbf{W}^{H} \mathbf{R}_{Y} \mathbf{W}}{\bar{S}} \int_{S}\left|\delta_{2}(\mathbf{r})\right|^{2} \mathrm{~d} S \\
& +2 \Re\left[\frac{\mathbf{W}^{H} \mathbf{r}_{Y L}}{\bar{S}} \int_{S} \delta_{2}(\mathbf{r}) \delta_{1}(\mathbf{r})^{*} \mathrm{~d} S\right] \\
& +\frac{\mathbf{R}_{L}}{\bar{S}} \int_{S}\left|\delta_{1}(\mathbf{r})\right|^{2} \mathrm{~d} S
\end{aligned}
$$

where $\mathbf{R}_{L}$ is the auto-correlation of the leakage signal $L$ :

$$
\mathbf{R}_{L}=\mathbb{E}\left\{|L|^{2}\right\}
$$

The FxMWF minimising (75) is then:

$$
\mathbf{W}_{\mathrm{ANC}}(S)=-\frac{\eta_{2,1}(S)}{\eta_{2,2}(S)} \mathbf{R}_{Y}^{-1} \mathbf{r}_{Y L}
$$

with

$$
\begin{aligned}
\eta_{2,2}(S) & \triangleq \frac{1}{S} \int_{S}\left|\delta_{2}(\mathbf{r})\right|^{2} \mathrm{~d} S \\
\eta_{2,1}(S) & \triangleq \frac{1}{S} \int_{S} \delta_{2}(\mathbf{r}) \delta_{1}(\mathbf{r})^{*} \mathrm{~d} S
\end{aligned}
$$

\section{Integrated active noise control and noise reduction}

In a similar way, a filter $\mathbf{W}_{\text {Int }}(\mathbf{S})$ may be defined corresponding to a desired ZQ $S$. This filter then minimises the aMSE criterion:

$$
\begin{aligned}
a J_{\text {Int }}(S) & =\frac{1}{\bar{S}} \int_{S} \mathbb{E}\left\{\left|E_{\text {Int }}(\mathbf{r})\right|^{2}\right\} \mathrm{d} S \\
E_{\text {Int }}(\mathbf{r}) & =C \cdot \mathbf{W}^{H} \mathbf{X}(\mathbf{r})+L^{n}(\mathbf{r})-D_{\mathrm{NR}}
\end{aligned}
$$

Assuming that the secondary path identification error is small $(\hat{C} \approx C)$, that the filter $\mathbf{W}$ is adapting slowly and that the noise and speech components of the microphone signals are uncorrelated, the aMSE criterion (83) can be written as:

$$
\begin{aligned}
a J_{\text {Int }}(S) & \approx \frac{1}{S} \int_{S} \mathbb{E}\left\{\left|\delta_{2}(\mathbf{r}) \mathbf{W}^{H} \mathbf{Y}^{s}-D_{\mathrm{NR}}\right|^{2} \mathrm{~d} S\right\} \\
+ & \frac{1}{S} \int_{S} \mathbb{E}\left\{\left|\delta_{2}(\mathbf{r}) \mathbf{W}^{H} \mathbf{Y}^{n}+\delta_{1}(\mathbf{r}) L^{n}\right|^{2} \mathrm{~d} S\right\}
\end{aligned}
$$

The integration being a linear operator, the aMSE can be rewritten as:

$$
\begin{aligned}
a J_{\mathrm{Int}}(S) \approx & \frac{\mathbf{W}^{H} \mathbf{R}_{Y} \mathbf{W}}{\bar{S}} \int_{S}\left|\delta_{2}(\mathbf{r})\right|^{2} \mathrm{~d} S \\
& +2 \Re\left[\frac{\mathbf{W}^{H} \mathbf{r}_{Y L^{n}}}{\bar{S}} \int_{S} \delta_{2}(\mathbf{r}) \delta_{1}(\mathbf{r})^{*} \mathrm{~d} S\right] \\
& -2 \Re\left[\frac{\mathbf{W}^{H} \mathbf{r}_{Y D_{\mathrm{NR}}}}{\bar{S}} \int_{S} \delta_{2}(\mathbf{r}) \mathrm{d} S\right] \\
& +\frac{\mathbf{R}_{L^{n}}}{\bar{S}} \int_{S}\left|\delta_{1}(\mathbf{r})\right|^{2} \mathrm{~d} S+|G|^{2} \mathbf{R}_{Y}
\end{aligned}
$$

Here $\mathbf{R}_{L^{n}}$ is the auto-correlation of the noise component of the leakage signal $L^{n}$ :

$$
\mathbf{R}_{L^{n}}=\mathbb{E}\left\{\left|L^{n}\right|^{2}\right\}
$$

The FxMWF minimising (74) is then:

$$
\mathbf{W}_{\text {Int }}(S)=\mathbf{R}_{Z Q}(S)^{-1} \mathbf{r}_{Z Q}(S)
$$

where

$$
\begin{aligned}
\mathbf{R}_{Z Q}(S) & =\eta_{2,2}(S) \mathbf{R}_{Y Y}(\omega) \\
\mathbf{r}_{Z Q}(S) & =\eta_{2}(S) \mathbf{r}_{Y^{s} D_{\mathrm{NR}}}-\eta_{2,1}(S) \mathbf{r}_{Y^{n} L^{n}}
\end{aligned}
$$

where $\eta_{2,2}(S)$ and $\eta_{2,1}(S)$ are defined in (81) and (82), respectively and $\eta_{2}(S)$ is defined as:

$$
\eta_{2}(S) \triangleq \frac{1}{S} \int_{S} \delta_{2}(\mathbf{r}) \mathrm{d} S
$$

\section{EXPERIMENTAL RESULTS}

The schemes introduced in Section III and Section IV have been tested experimentally for both ANC and integrated ANC and NR and their performance has been compared with the performance of a standard ANC scheme and the original integrated ANC and NR scheme [12] described in Section II, respectively.

\section{A. Experimental setup}

The simulations were run on acoustic path measurements obtained with a CORTEX MK2 manikin head and torso equipped with artificial ears and a two-microphones behindthe-ear (BTE) hearing aid. The sound sources (FOSTEX 6301B loudspeakers) were positioned at 1 meter from the centre of the head. The speech source was located at $0^{\circ}$ and the noise source at $270^{\circ}$. The BTE was worn on the left ear, facing the noise source at $270^{\circ}$. Commercial hearing aids currently do not have an ear canal microphone, therefore the artificial ear simulator is used here as ear canal microphone to generate the error signal. The tests were run on 22 seconds long signals. The noise was a multitalker babble from Auditec [29]. The speech was composed of three sentences from the HINT database [30] concatenated with silence periods. All the signals were sampled at $16 \mathrm{kHz}$.

In these experiments, the ear canal microphone was considered to be located $15 \mathrm{~mm}$ away from the eardrum. The hearing aid loudspeaker is located $20 \mathrm{~mm}$ away from the ear canal microphone and the angle $\alpha$ is set to $\frac{5 \pi}{6}$ (as shown in Figure 6).

The MWF length is set to $N=128$, and, in the case of the integrated ANC and NR schemes, the NR delay is set to half the filter length $(\Delta=64)$. The secondary path $C$ is estimated off-line using an identification technique based on the Normalised-Least-Mean-Squares (NLMS) algorithm. The length of the estimated path $\hat{C} s$ is set to $L=32$.

The RP-based schemes (ANC and integrated ANC and NR) are designed to optimise the MSE criterion at the central point of the eardrum (point $\mathbf{r}_{\mathrm{TM}}$ in Figure 6).

In the case of ZQ-based schemes (ANC and integrated ANC and NR schemes), two different zones are considered, the first zone (zone 1 in Figure 6) is a section of the portion of the ear canal between the ear canal microphone and the eardrum (considered to be a $15 \mathrm{~mm}$ by $10 \mathrm{~mm}$ rectangular zone), the second zone (zone 2 in Figure 6) is a section of the close neighbourhood of the eardrum (considered to be a $5 \mathrm{~mm}$ by $10 \mathrm{~mm}$ rectangular zone).

The performance measure used for the ANC schemes is the residual noise power at the eardrum. The reference power 


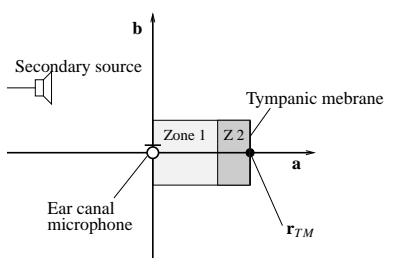

Fig. 6. Simulation setup.

is the power of the leakage signal's noise component, which can also be considered as the residual noise power when the hearing aid is turned off. Note that it can be shown from (36) that the power of the leakage signal's noise component is stationary in space, i.e., in the ear canal. The performance measure is then written as:

$$
\Delta \operatorname{Pow}^{n}(\mathbf{r})=\operatorname{Pow}_{\text {out }}^{n}(\mathbf{r})-\operatorname{Pow}_{\text {leak }}^{n}
$$

where $\operatorname{Pow}_{\text {out }}^{n}(\mathbf{r})$ and $\operatorname{Pow}_{\text {leak }}^{n}$ are the power (in $\mathrm{dB}$ ) of the residual noise at point $\mathbf{r}$ and the power of the noise component of the leakage signal, respectively.

$$
\begin{aligned}
\operatorname{Pow}_{\text {out }}^{n}(\mathbf{r}) & =\mathbb{E}\left\{\left|\mathbf{W}^{H} \mathbf{X}^{n}(\mathbf{r})+L^{n}(\mathbf{r})\right|^{2}\right\} \\
\text { Pow }_{\text {leak }}^{n} & =\mathbb{E}\left\{\left|L^{n}\right|^{2}\right\}
\end{aligned}
$$

The performance measure used for integrated ANC and NR schemes is the intelligibility-weighted SNR. Evaluation of NR in hearing aids can rely on a variety of performance measures and most commonly perceptual measures such as the speech reception threshold (SRT), listening effort scaling or preference rating. Evaluating perceptual measures requires listening tests which were not carried out during this work. The intelligibility-weighted SNR, however, has been shown to be correlated with the SRT and is therefore used here as a performance measure.

The leakage SNR, which can also be considered as the SNR when the hearing aid is turned off, is used as a reference measure. Note that it can be shown from (36) that the SNR of the leakage signal is stationary in space, i.e., in the ear canal. The intelligibility-weighted SNR improvement [31] is defined as:

$$
\Delta \mathrm{SNR}_{\text {intellig }}(\mathbf{r})=\sum_{i} I_{i}\left(\mathrm{SNR}_{i, \text { out }}(\mathbf{r})-\mathrm{SNR}_{i, \text { leak }}\right)
$$

for the RP-based schemes, and:

$$
a \Delta \mathrm{SNR}_{\text {intellig }}=\frac{1}{S} \int_{S} \Delta \mathrm{SNR}_{\text {intellig }}(\mathbf{r}) \mathrm{d} S
$$

for the ZQ-based schemes.

Here $I_{i}$ is the band importance function and $\mathrm{SNR}_{i \text {,out }}(\mathbf{r})$ and $\mathrm{SNR}_{i \text {, leak }}$ represent the output SNR at point $\mathbf{r}$ and the leakage SNR (in $\mathrm{dB}$ ) of the $i$ th band, respectively.

At this stage, the signals at distant point (r) are obtained based on acoustic propagation models. A final validation of the algorithm would, however, require sound pressure measurements along the ear canal but those are not the focus of this paper.

\section{B. Active noise control}

The residual noise power has been computed in the ear canal and the performance is presented for a standard ANC scheme

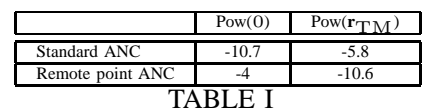

NOISE POWER AT THE EAR CANAL MICROPHONE AND AT THE EARDRUM (IN DB), $\delta=3$.

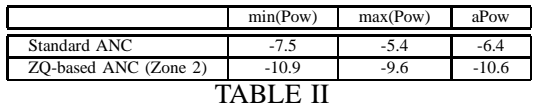

MINIMUM, MAXIMUM AND AVERAGE NOISE POWER OVER ZONE 2 (IN DB), $\delta=3$.

(Figure 7(a)), a RP-based ANC scheme designed to minimise the noise at point $\mathbf{r}_{\mathrm{TM}}$ (Figure 7(b)), a ZQ-based ANC scheme designed to minimise the noise power over zone 1 (Figure 7(c)) and a ZQ-based ANC scheme defined to minimise the noise power over zone 2 (Figure $7(\mathrm{~d})$ ). All these schemes are based on a FxMWF. For the first set of results presented here the hearing aid delays are neglected $\left(\Delta_{\mathrm{HA}}=0\right)$ resulting in a causality margin $\delta=3$.

The standard ANC scheme allows to reduce the noise power by about $10 \mathrm{~dB}$ at the ear canal microphone but its performance is degraded to a reduction of $4 \mathrm{~dB}$ at the eardrum $\left(\mathbf{r}_{\mathrm{TM}}\right)$. The RP-based ANC scheme allows to reduce the noise power by about $10 \mathrm{~dB}$ at $\mathbf{r}_{\mathrm{TM}}$ and therefore restores the ANC performance at the eardrum (Table I). When the noise is to be controlled over a desired zone-of-quiet, however, the RPbased ANC scheme does not provide a satisfying approach as its residual noise power quickly increase when moving away from $\mathbf{r}_{\mathrm{TM}}$.

The noise reduction performance of the ZQ-based ANC designed to minimise the noise over the close neighbourhood of the eardrum (zone 2) is shown in Figure 7(d). When the standard ANC scheme achieves an average noise reduction of $6 \mathrm{~dB}$ over the considered zone, the ZQ-based ANC scheme achieves an average performance of about $10 \mathrm{~dB}$. The maximum noise power over the considered zone then drops from $-5.4 \mathrm{~dB}$ with the standard ANC scheme to $-9.6 \mathrm{~dB}$ with the ZQ-based ANC scheme (Table II). The risk of constructive interferences is then highly reduced.

When the noise is controlled over the full ear canal (zone 1), the average noise reduction delivered by the standard ANC scheme is $8 \mathrm{~dB}$. The ZQ-based ANC scheme set to minimise the noise over zone 1 (Figure 7(c)) achieves an average noise reduction of about $10 \mathrm{~dB}$ improving the performance of the standard scheme by almost $2 \mathrm{~dB}$. The maximum noise power over the ear canal also drops from $-5.4 \mathrm{~dB}$ with the standard ANC scheme to $-7.4 \mathrm{~dB}$ with the ZQ-based ANC scheme (Table III).

It appears that for the standard ANC scheme the noise reduction performance quickly decreases when the ear canal microphone is away from the eardrum. The RP-based approach allows to restore the ANC performance at a single point but its performance also decreases when moving away from the optimised point. When the noise is to be considered over a zone-of-quiet, the ZQ-based approach has shown to provide significant performance improvement. It also allows to keep the maximum noise power over the zone-of-quiet at a lower level than with the standard ANC scheme, thus preventing for possible constructive interferences. 


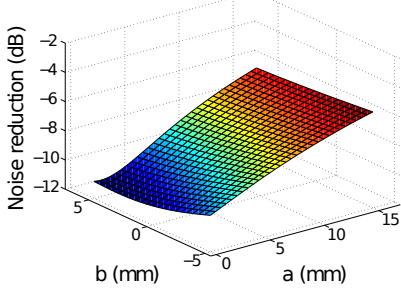

(a) Standard Scheme

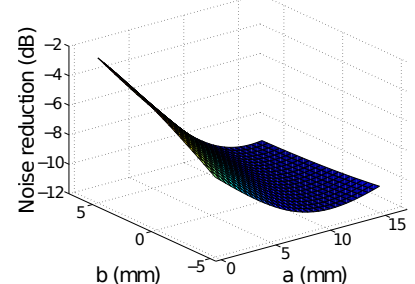

(b) Remote point

Fig. 7. Noise reduction for multichannel ANC, $\delta=3$

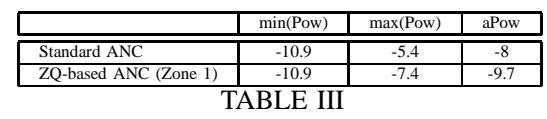

MINIMUM, MAXIMUM AND AVERAGE NOISE POWER OVER ZONE 1 (IN $\mathrm{DB}), \delta=3$.

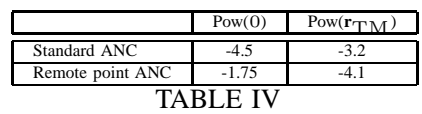

NOISE POWER AT THE EAR CANAL MICROPHONE AND AT THE EARDRUM (IN DB), $\delta=-1$.

The performance detailed above is obtained for a sufficiently high causality margin $(\delta=3)$. It is important, however, to keep in mind that the causality can greatly affect the performance of any ANC-based scheme. Therefore, Tables IV-VI present the performance of the standard ANC scheme, the RP-based ANC scheme and the ZQ-based ANC scheme when the system is slightly non-causal $(\delta=-1)$. Tables VII-IX present the performance of the standard ANC scheme, the RP-based ANC scheme and the ZQ-based ANC scheme when the system is highly non-causal $(\delta=-10)$.

When the ANC has to operate on a setup with a negative causality margin $(\delta=-1)$, the noise power reduction performance at the ear canal microphone is degraded from about $10 \mathrm{~dB}$ (with $\delta=3$ ) to $4.5 \mathrm{~dB}$. This performance is reduced furthermore at the eardrum to a noise power reduction of about $3 \mathrm{~dB}$. The RP-based approach allows to almost fully restore this performance at the eardrum while the ZQ-based ANC scheme allow to restore about $0.5 \mathrm{~dB}$ to $1 \mathrm{~dB}$ of the average noise reduction over the desired $\mathrm{ZQ}$, depending on the $\mathrm{ZQ}$ considered. When the causality margin is slightly negative $(\delta=-1)$ the performance of the ANC schemes is degraded but the behaviour of the RP-based scheme and the ZQ-based scheme stays the same as when operating on a causal setup.

When the causality margin is further reduced $(\delta=-10)$ the performance of the standard ANC scheme is degraded and any change due to the RP-based scheme or the ZQ-based schemes is insignificant.

\section{Integrated active noise control and noise reduction}

The SNR has been computed over the ear canal and the aSNR has been computed over the two zones of quiet for the output of the original integrated ANC and NR scheme the RP-based integrated ANC and NR scheme designed to minimise the MSE criterion (51) at point $\mathbf{r}_{\mathrm{TM}}$, a ZQ-based integrated ANC and NR scheme designed to minimise the aMSE criterion (83) over the ear canal (zone 1) and a ZQbased integrated ANC and NR scheme designed to minimise the aMSE criterion over the neighbourhood of the eardrum (zone 2). It has been shown in the previous section that, with a

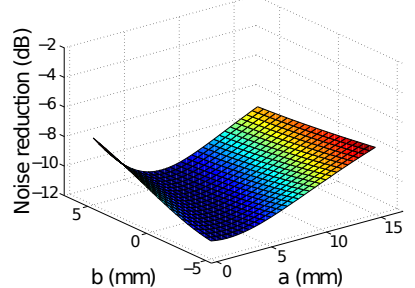

(c) ZQ-based ANC (Zone 1)

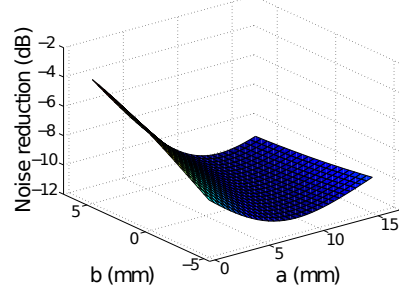

(d) ZQ-based ANC (Zone 2)

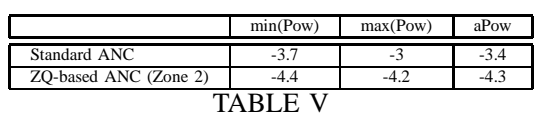

MINIMUM, MAXIMUM AND AVERAGE NOISE POWER OVER ZONE 2 (IN DB), $\delta=-1$.

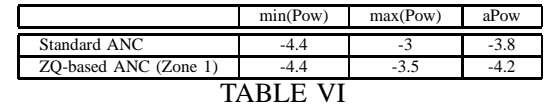

MINIMUM, MAXIMUM AND AVERAGE NOISE POWER OVER ZONE 1 (IN DB), $\delta=-1$.

negative causality margin, the performance of the ANC-based schemes is degraded. Therefore, the results presented in this section are obtained while neglecting the hearing aid delays $\left(\Delta_{\mathrm{HA}}=0\right)$, i.e., the causality margin $\delta=3$. A more complete study of the impact of the causality on the performance of the integrated ANC and NR scheme can be found in [12].

The original integrated ANC and NR scheme delivers a SNR of about $9 \mathrm{~dB}$ at the ear canal microphone but its performance is fluctuating over the ear canal (Figure 9(a)) and its output SNR eventually decreases to $4.4 \mathrm{~dB}$ at the eardrum $\left(\mathbf{r}_{\mathrm{TM}}\right)$. The RP-based integrated ANC and NR scheme delivers a $9.7 \mathrm{~dB}$ SNR at $\mathbf{r}_{\mathrm{TM}}$ and therefore restores the original integrated ANC and NR scheme performance at the eardrum (Table X). When the noise is to be controlled over a desired zone-of-quiet however, the RP-based integrated ANC and NR scheme does not provide a satisfying approach as its SNR quickly decreases as moving away from $\mathbf{r}_{\mathrm{TM}}$.

The SNR performance of the ZQ-based integrated ANC and NR scheme designed to minimise the noise over the close neighbourhood of the eardrum (zone 2) is shown in Figure 8(b) and compared to the performance of the original integrated ANC and NR scheme over the same zone (Figure 8(a)). When the original integrated ANC and NR scheme achieves an aSNR of $4.9 \mathrm{~dB}$ over the considered zone, the ZQ-based integrated ANC and NR scheme achieves an aSNR of about $9 \mathrm{~dB}$. The minimum SNR over the zone then increases from $4.1 \mathrm{~dB}$ with the original integrated ANC and NR scheme to about $8 \mathrm{~dB}$ with the ZQ-based integrated ANC and NR scheme (Table II).

When the zone-of-quiet to be considered is the full ear canal (zone 1) the aSNR (Figure 9(a)) delivered by the original integrated ANC and NR scheme is $6.4 \mathrm{~dB}$. The ZQ-based integrated ANC and NR scheme designed to minimise the aMSE over zone 1 (Figure 9(b)) delivers an aSNR of 8.3dB. The minimum SNR over the ear canal then increases from $4.1 \mathrm{~dB}$ with the original ANC and NR scheme to about $5.9 \mathrm{~dB}$ with the ZQ-based integrated ANC and NR scheme (Table II).

It appears that the performance of the original integrated ANC and NR scheme quickly decreases when the ear canal 


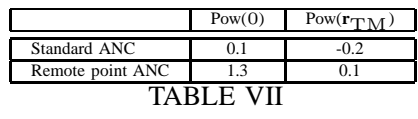

TABLE VII

NOISE POWER AT THE EAR CANAL MICROPHONE AND AT THE EARDRUM (IN DB), $\delta=-10$.

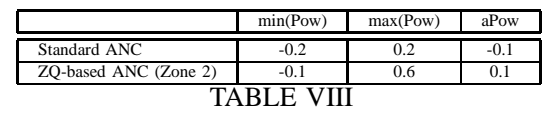

MINIMUM, MAXIMUM AND AVERAGE NOISE POWER OVER ZONE 2 (IN DB), $\delta=-10$.

microphone is away from the eardrum. The RP-based approach allows to restore the integrated ANC and NR scheme's performance at a single point but its performance also decrease when moving away from the optimised point. The ZQ-based approach, adjusted to the zone on which the noise is to be cancelled, exhibits improved performance in aSNR compared to the original integrated ANC and NR scheme. It also allows to improve the minimum SNR, therefore reducing the impact of the performance degradation where it is most significant.

\section{CONCLUSION}

It has been shown in the previous work that ANC provides an efficient solution to the signal leakage problem in hearing aids with an open fitting. The integrated ANC and NR scheme allows to control the noise at the ear canal microphone. Ideally, this microphone should be located at the eardrum. In practice however, the ear canal microphone is away from the eardrum and hence the sound reaching the eardrum is basically unknown and uncontrolled.

The RP-based approach presented here is based on an MSE criterion expressed at a RP away from the ear canal microphone. The RP-based approach applied to the ANC scheme and the integrated ANC and NR scheme, has been shown to restore the performance of the original schemes at the RP. Besides, the structure of the filters that are derived based on the RP-based approach is very similar to the well-known Filtered-X structure. Under a single speech source scenario and when the number of sound sources (speech source plus noise sources) is less than or equal to the number of hearing aid microphones, it is possible to derive a formula for the RP-based integrated ANC and NR SNR performance. This confirms that the RP-based approach allows to restore at an RP the integrated ANC and NR scheme SNR performance at the ear canal microphone. The RP-based approach, however, cannot be a satisfying approach when the noise is to be controlled over a ZQ rather than at a single point.

The so-called ZQ-based approach allows to optimise the average of a particular design criterion, here the MSE criterion, over a ZQ generated by the ANC. This approach, applied to the ANC scheme and the integrated ANC and NR scheme, exhibits improved performance, compared to the original schemes, especially when the noise is to be canceled on a zone that is away from the ear canal microphone. The ZQ-based approach also allows to reduce the impact of constructive interference over the desired $Z Q$.

The ZQ-based approach thus provides a more robust and more realistic way to apply ANC and solve the signal leakage problem in the framework of hearing aids with an open fitting.

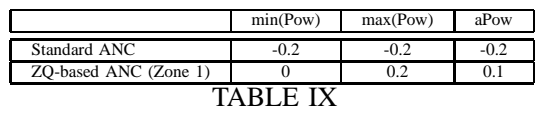

MINIMUM, MAXIMUM AND AVERAGE NOISE POWER OVER ZONE 1 (IN DB), $\delta=-10$

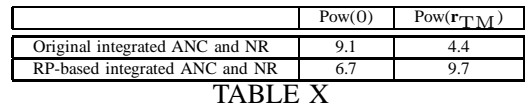

SNR PERFORMANCE AT THE EAR CANAL MICROPHONE AND AT THE EARDRUM (IN DB), $\delta=3$.

\section{REFERENCES}

[1] P. C. Loizou, Speech Enhancement: Theory and Practice. Boca Raton, FL: CRC Press, 2007.

[2] L. J. Griffiths and C. W. Jim, "An alternative approach to linearly constrained adaptive beamforming," IEEE Transactions on Antennas and Propagation, vol. 30, pp. 27-34, 1982.

[3] S. Doclo, A. Spriet, J. Wouters, and M. Moonen, "Frequency-domain criterion for the speech distortion weighted multichannel wiener filter for robust noise reduction," Elsevier Speech Communication, vol. 49, no. 7-8, pp. 636-656, 2007.

[4] T. van Waterschoot, "Design and evaluation of digital signal processing algorithms for acoustic feedback and echo cancellation," Ph.D. dissertation, Katholieke Universiteit Leuven, Leuven, Belgium, Mar. 2009.

[5] K. Ngo, T. van Waterschoot, M. G. Christensen, M. Moonen, S. H. Jensen, and J. Wouters, "Adaptive feedback cancellation in hearing aids using a sinusoidal near-end signal model," in IEEE International Conference on Acoustics, Speech and Signal Processing, (ICASSP'10), Dallas, Texas, USA, Mar. 2010.

[6] J. Kiessling, "Sounds towards the tympanic membrane," in 8th EFAS Congress. Heidelberg: European Federation of Audiological Societies, June 2007.

[7] S. J. Elliott and P. A. Nelson, active control of sound. Cambridge: Academic press, 1993.

[8] S. M. Kuo and D. R. Morgan, "Active noise control: a tutorial review," Proceedings of the IEEE, vol. 87, Issue: 6, no. 0018-9219, pp. 943973, Jun 1999.

[9] E. Bjarnason, "Analysis of the filtered-X LMS algorithm," IEEE Transactions on Speech and Audio Processing, vol. 3, no. 6, pp. 504-514, 1995.

[10] J. C. Burgess, "Active adaptive sound control in a duct: a computer simulation," The Journal of the Acoustical Society of America, vol. 70, Issue 3, pp. 715-726 715-726, September 1981.

[11] B. Widrow and S. D. Stearns, Adaptive signal processing. Englewood Cliffs,N J: Prentice-Hall, 1985.

[12] R. Serizel, M. Moonen, J. Wouters, and S. H. Jensen, "Integrated active noise control and noise reduction in hearing aids," IEEE Transactions on Speech Audio and Language, vol. 18, no. 6, pp. 1137-1146, August 2010.

[13] P. A. Nelson, J. K. Hammond, P. Joseph, and S. J. Elliott, “Active control of stationary random sound fields," The Journal of the Acoustical Society of America, vol. 87, p. 963, 1990.

[14] P. Joseph, S. J. Elliott, and P. A. Nelson, "Near field zones of quiet," Journal of Sound and Vibration, vol. 172, issue 5, pp. 605-627, may 1994.

[15] B. Rafaely, "Zones of quiet in a broadband diffuse sound field," The Journal of the Acoustical Society of America, vol. 110, p. 296, 2001.

[16] R. Serizel, M. Moonen, J. Wouters, and S. H. Jensen, "A zone of quiet based approach to integrated active noise control and noise reduction in hearing aids," in The 2009 IEEE Workshop on Applications of Signal Processing to Audio and Acoustics (WASPAA 2009), October 2009.

[17] — , "Output snr analysis of integrated active noise control and noise reduction in hearing aids under a single speech source," EURASIP Signal Processing, vol. 91, no. 8, pp. 1719-1729, August 2011.

[18] H. Dillon, Hearing Aids. Thieme, 2001.

[19] J. M. Kates, Digital hearing aids. Cambridge Univ Press, 2008.

[20] X. Kong and S. M. Kuo, "Study of causality constraint on feedforward active noise controlsystems," IEEE Transactions on Circuits and Systems II: Analog and Digital Signal Processing, vol. 46, no. 2, pp. 183-186, 1999.

[21] D. R. Morgan and J. C. Thi, "A delayless subband adaptive filter architecture," IEEE Transactions on Signal Processing, vol. 43, no. 8, pp. 1819-1830, 1995. 


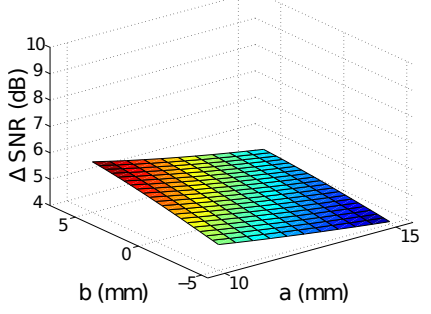

(a) Original scheme

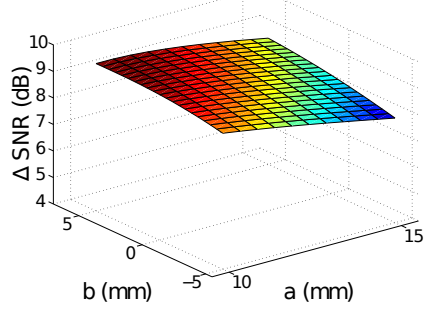

(b) ZQ-based scheme (Zone 2)
Fig. 8. SNR performance of integrated ANC and NR schemes over zone 2, $\delta=3$

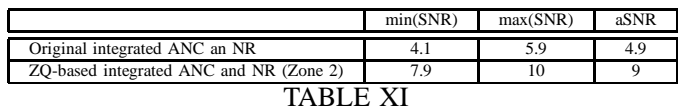

Minimum AND AVERAGE SNR, OVER ZONE 2 (IN DB), $\delta=3$.

[22] B. Rafaely and M. Furst, "Audiometric ear canal probe with active ambient noise control," IEEE Transactions on Speech and Audio Processing, vol. 4, Issue 3, no. 1063-6676, pp. 224-230, May 1996.

[23] S. Doclo and M. Moonen, "GSVD-based optimal filtering for single and multimicrophone speech enhancement," IEEE Transactions on Signal Processing, vol. 50, no. 9, pp. 2230-2244, 2002.

[24] S. Haykin, Adaptive Filter Theory. Fourth Edition. Prentice Hall, 2002.

[25] L. J. Eriksson and M. C. Allie, "Use of random noise for on-line transducer modeling in an adaptive active attenuation system," The Journal of the Acoustical Society of America, vol. 85, no. 2, pp. 797 802, February 1989.

[26] S. M. Kuo and D. Vijayan, "A secondary path modeling technique for active noise control systems," IEEE Transactions on Speech and Audio Processing, vol. 5, no. 4, pp. 374-377, 1997.

[27] M. Zhang, H. Lan, and W. Ser, "Cross-updated active noise control system with online secondary path modeling," IEEE Transactions on Speech and Audio Processing, vol. 9, no. 5, pp. 598-602, 2001.

[28] B. Rafaely, "Spatial-temporal correlation of a diffuse sound field," The Journal of the Acoustical Society of America, vol. 107, pp. 3254-3258, 2000.

[29] Auditec, "Auditory Tests (Revised), Compact Disc, Auditec, St. Louis," St. Louis, 1997.

[30] M. Nilsson, S. D. Soli, and A. Sullivan, "Development of the Hearing in Noise Test for the measurement of speech reception thresholds in quiet and in noise," The Journal of the Acoustical Society of America, vol. 95, no. 2, pp. 1085-1099, Feb. 1994.

[31] J. E. Greenberg, P. M. Peterson, and P. M. Zurek, "Intelligibilityweighted measures of speech-to-interference ratio and speech system performance," The Journal of the Acoustical Society of America, vol. 94, no. 5, pp. 3009-3010, Nov. 1993.

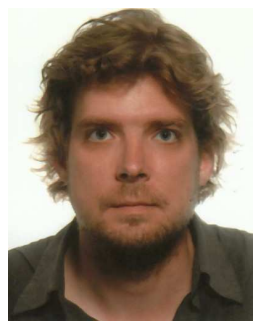

Romain Serizel Romain Serizel received the M.Eng degree in Automatic System Engineering from ENSEM (Nancy, France) in 2005 and the M.Sc. degree in Signal Processing from Universit Rennes 1 (Rennes, France) in 2006. He received the Ph.D. degree in Engineering Sciences from the Katholieke Universiteit Leuven (KUL), Belgium in June 2011.

Since 2011 he is a research assistant with Prof Marc Moonen research group at the Electrical Engineering Department (ESAT-SCD) of the KUL. His research interests include hearing aids systems, cochlear implants and digital signal processing for audio.

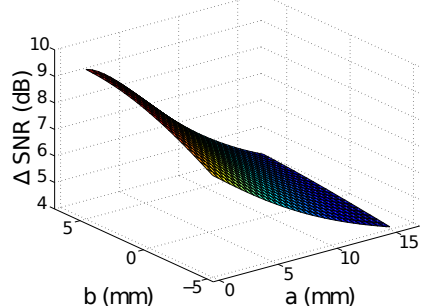

(a) Original scheme

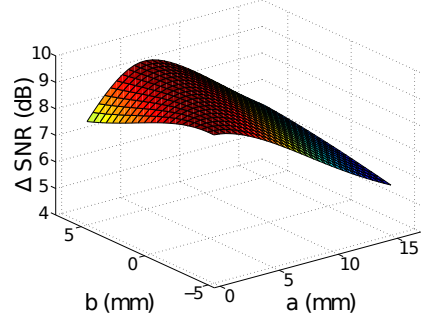

(b) ZQ-based scheme (Zone 1)
Fig. 9. SNR performance of integrated ANC and NR schemes over zone 1, $\delta=3$

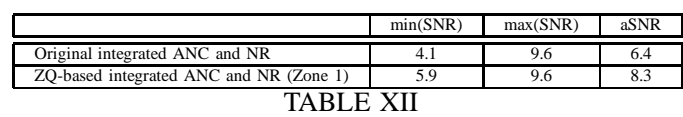

Minimum AND AVERAGE SNR, OVER ZONE 1 (IN DB), $\delta=3$.

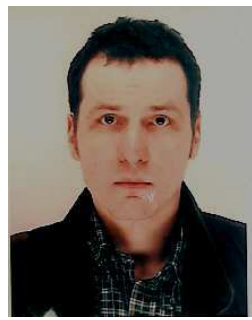

Marc Moonen Marc Moonen (M'94, SM'06, F'07) received the electrical engineering degree and the $\mathrm{PhD}$ degree in applied sciences from Katholieke Universiteit Leuven, Belgium, in 1986 and 1990 respectively.

Since 2004 he is a Full Professor at the Electrical Engineering Department of Katholieke Universiteit Leuven, where he is heading a research team working in the area of numerical algorithms and signal processing for digital communications, wireless communications, DSL and audio signal processing.

He received the 1994 K.U.Leuven Research Council Award, the 1997 Alcatel Bell (Belgium) Award (with Piet Vandaele), the 2004 Alcatel Bell (Belgium) Award (with Raphael Cendrillon), and was a 1997 "Laureate of the Belgium Royal Academy of Science". He received a journal best paper award from the IEEE Transactions on Signal Processing (with Geert Leus) and from Elsevier Signal Processing (with Simon Doclo).

He was chairman of the IEEE Benelux Signal Processing Chapter (19982002), and is currently Past-President of EURASIP (European Association for Signal Processing) and a member of the IEEE Signal Processing Society Technical Committee on Signal Processing for Communications.

He has served as Editor-in-Chief for the "EURASIP Journal on Applied Signal Processing" (2003-2005), and has been a member of the editorial board of "IEEE Transactions on Circuits and Systems II" (2002-2003) and "IEEE Signal Processing Magazine" (2003-2005) and "Integration, the VLSI Journal". He is currently a member of the editorial board of "EURASIP Journal on Applied Signal Processing", "EURASIP Journal on Wireless Communications and Networking", and "Signal Processing".

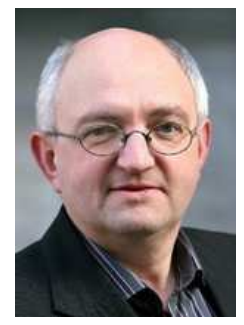

Jan Wouters Jan Wouters was born in Leuven, Belgium, in 1960. He received the physics degree and the Ph.D. degree in sciences / physics from the Katholieke Universiteit Leuven, Leuven, Belgium, in 1982 and 1989, respectively. From 1989 till 1992 he was a Research Fellow with the Belgian National Fund for Scientific Research (FWO) at the Institute of Nuclear Physics (UCL Louvain-la-Neuve and K.U.Leuven) and at NASA Goddard Space Flight Center (USA). Since 1993 he is a Professor at the Neurosciences Department of the K.U.Leuven (Full Professor since 2001). His research activities center around audiology and the auditory system, signal processing for cochlear implants and hearing aids. $\mathrm{He}$ is author of about 145 articles in international peer-reviewed journals and is a reviewer for several international journals.

Dr. Wouters received an Award of the Flemish Ministery in 1989, a Fullbright Award and a NATO Research Fellowship in 1992, and the Flemish VVL Speech therapy - Audiology Award in 1996. He is member of the International Collegium for ORL (CORLAS), a Board Member of the International Collegium for Rehabilitative Audiology (ICRA) and is responsible for the Laboratory for Experimental ORL and the audiology program at K.U.Leuven. 


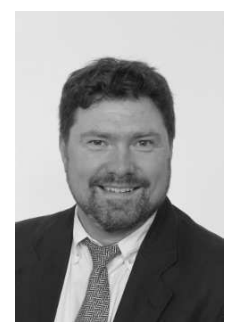

Søren Holdt Jensen Sren Holdt Jensen (S87M88SM00) received the M.Sc. degree in electrical engineering from Aalborg University, Aalborg, Denmark, in 1988, and the Ph.D. degree in signal processing from the Technical University of Denmark, Lyngby, Denmark, in 1995. Before joining the Department of Electronic Systems of Aalborg University, he was with the Telecommunications Laboratory of Telecom Denmark, Ltd, Copenhagen, Denmark; the Electronics Institute of the Technical University of Denmark; the Scientific Computing Group of Danish Computing Center for Research and Education (UNI•C), Lyngby; the Electrical Engineering Department of Katholieke Universiteit Leuven, Leuven, Belgium; and the Center for PersonKommunikation (CPK) of Aalborg University.

$\mathrm{He}$ is Full Professor and is currently heading a research team working in the area of numerical algorithms and signal processing for speech and audio processing, image and video processing, multimedia technologies, and digital communications.

Prof. Jensen was an Associate Editor for the IEEE Transactions on Signal Processing and Elsevier Signal Processing, and is currently Member of the Editorial Board of IEEE Transactions on Audio, Speech and Language Processing and EURASIP Journal on Advances in Signal Processing. He is a recipient of an European Community Marie Curie Fellowship, former Chairman of the IEEE Denmark Section, and Founder and Chairman of the IEEE Denmark Sections Signal Processing Chapter. He is member of the Danish Academy of Technical Sciences and was in January 2011 appointed as member of the Danish Council for Independent Research-Technology and Production Sciences by the Danish Minister for Science, Technology and Innovation. 